\title{
Excimers from Stable and Persistent Supramolecular Radical-Pairs in Red/NIR- Emitting Organic Nanoparticles and Polymeric Films
}

\author{
Davide Blasi, Domna M. Nikolaidou, Francesca Terenziani, Imma Ratera, and Jaume Veciana
}

In this work, the luminescence properties of new materials based on open-shell molecular systems are studied. In particular, we prepared polymeric films and organic nanoparticles (ONPs) doped with triphenylmethyl radical molecules. ONPs exhibit uniform size distribution, spherical morphology and high colloidal stability. The emission spectrum of low-doped ONP suspensions and low-doped films is very similar to the emission spectrum of TTM in solution, while the luminescence lifetime and the luminescence quantum yield (LQY) are highly increased. Increasing the radical doping leads to a progressive decrease of the LQY and the appearance of a new broad excimeric band at longer wavelengths, both for ONPs and films. Thus, not only the luminescence properties were improved, but also the formation of excimers from stable and persistent supramolecular radical-pairs was observed for the first time. The good stability and luminescence properties with emission in the red-NIR region (650-800 nm), together with the open-shell nature of the emitter, make these free-radical excimer-forming materials promising candidates for optoelectronics and bioimaging applications.

\section{Introduction}

The Polychlorotriphenylmethyl (PTM) radicals are considered inert carbon-centered free-radicals thanks to their high chemical and thermal stability both in solution and in solid state, where their half-life is on the order of decades. ${ }^{1}$ This stability, joined with the unusual physical and chemical properties given by the open-shell electronic configuration, offers the possibility to exploit these molecules in different fields, ${ }^{2}$ with remarkable applications as molecular switches, ${ }^{3,4}$ molecular wires, ${ }^{5,6}$ mediators in $\mathrm{Li} / \mathrm{O}_{2}$ batteries ${ }^{7}$ or as neutral molecular conductors. ${ }^{8}$ Recently, rising interest has been devoted not only to the electronic and magnetic behaviour of open-shell materials, but also to their optical and optoelectronic properties. ${ }^{9-12}$ Indeed, the presence of an unpaired electron mostly localized on the $\alpha$-carbon, is responsible for several peculiar characteristics: a doublet electronic configuration, emission at long wavelengths despite the absence of elongated $\pi$-systems, large Stokes shift and emission lifetimes on the order of tens of ns. ${ }^{13-16}$ Very recently Peng et al. have reported on the fabrication of the first OLED based on neutral $\pi$ radicals, ${ }^{17}$ in which the use of a doublet emitter, avoiding the formation of singlet and triplet excitons, ${ }^{18}$ can rise the upper limit of internal quantum efficiency to $100 \%$. However, there are several limiting factors for the use of these radical molecules as photoactive species in optoelectronic devices or as luminescent probes for bioimaging applications. For example, their almost complete insolubility in water hinders their use in biological applications. Another limiting factor is the typically low value of luminescence quantum yield (LQY). In fact, there are only few examples in which PTM radical derivatives show a LQY higher than 0.3 and only in apolar or poorly polar solvents, ${ }^{10,12}$ while in the solid state the emission is completely quenched. Moreover, photostability is also a limiting issue, particularly in solution where, for example, the perchlorotriphenylmethyl radical shows a photoreaction quantum yield of $0.3 .^{19}$ In order to overcome these drawbacks, Hattori et al. have recently proposed different molecular strategies, like the introduction of heteroatoms in the phenyl rings, the formation of coordination complexes or the substitution of the halogen atoms in the ortho positions. ${ }^{20-23}$ These functionalizations allowed to reach values of photostability in solution hundred times higher than the one of the tris(2,4,6-trichlorophenyl)methyl radical (TTM) (Figure 1), one of the simplest and most studied inert carbon-centered free-radicals.

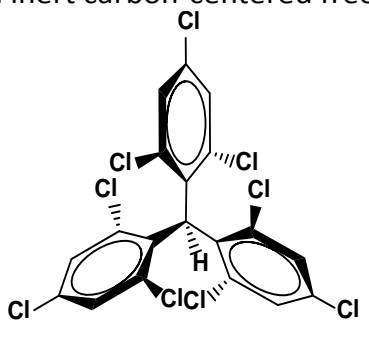

TTM- $\alpha \mathrm{H}$

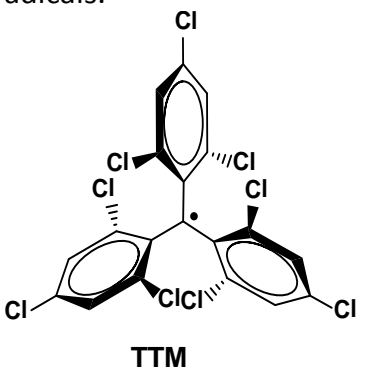

TTM

Fig. 1. Chemical structure of TTM- $\alpha \mathrm{H}$ (ONPs host) and TTM radical (ONPs guest).

A few studies on self-assembled monolayers ${ }^{24,25}$ or micro-particles ${ }^{26}$ of PTM radicals have also been reported but, to the best of our knowledge, no systematic studies are available on the confinement and nanostructuration of radical molecules in solid organic matrices such as organic nanoparticles. Nonetheless, interesting effects can be expected because of reduced vibrations and intermolecular interactions responsible for non-radiative decays. ${ }^{27}$ On the other hand, the formation in the solid state of emissive supramolecular aggregates like excimers is a very interesting and exploited strategy in order to have a wide emission 
able to cover the NIR region, ${ }^{28-32}$ but up to now, no examples of excimers based on stable and persistent radical pairs have been reported. Here, we present a systematic study of the optical properties of an open-shell radical molecule (TTM, Figure 1) dispersed into organic nanoparticles (ONPs) and in polymeric films. Specifically, we investigate the optical behavior of ONPs made by the closed-shell and optically neutral tris(2,4,6-trichlorophenyl)methane (TTM- $\alpha \mathrm{H})$ (Figure 1), as well as PMMA films, both doped with different amounts of TTM.

\section{Results and Discussion}

The absorption and luminescence spectra and the LQY of TTM dissolved in THF (solvent used for the preparation of ONPs) (Figure S1 and Table 1) and in glassy 2-methyl THF at $77 \mathrm{~K}$ (Figure S2 and Table 1) were measured, as reference starting points. In the undercooled solution, the radical exhibits a LQY of 0.72 , more than 35 times higher than in THF, indicating that the confinement in a rigid host can improve the luminescence.

Table 1. Optical properties of TTM radical in THF at room temperature and in glassy 2-methyl THF at $77 \mathrm{~K}$.

\begin{tabular}{|c|c|c|c|c|c|c|c|}
\hline & $\begin{array}{l}\lambda_{\mathrm{abs}}{ }^{\max } \\
{[\mathrm{nm}]}\end{array}$ & $\begin{array}{l}\lambda_{\mathrm{em}}{ }^{\max } \\
{[\mathrm{nm}]}\end{array}$ & $\begin{array}{c}\text { Stokes shift } \\
{\left[\mathrm{cm}^{-1}\right]}\end{array}$ & $\begin{array}{c}\varepsilon \\
{\left[\mathrm{cm}^{-1} \mathrm{M}^{-1}\right]} \\
\end{array}$ & $\operatorname{LQY}^{\text {a) }}$ & $\begin{array}{l}\tau^{\mathrm{b})} \\
{[\mathrm{s}]}\end{array}$ & $\begin{array}{l}\text { a) Luminescence Quantum Yields. b) Fluorescence lifetime estimated through } \\
\text { mono-exponential reconvolution fit analysis. }\end{array}$ \\
\hline $\begin{array}{l}\text { THF } \\
\text { (room T) }\end{array}$ & 372 & 566 & 9214 & 36840 & 0.02 & $7.6 \times 10^{-9}$ & Since pure TTM radical ONPs undergo almost complete \\
\hline $\begin{array}{l}\text { 2-MeTHF } \\
\text { (77 K) }\end{array}$ & 375 & 556 & 8681 & - & 0.72 & - & $\begin{array}{l}\text { aggregation-caused quenching (ACQ) of fluorescence, aqueous } \\
\text { suspensions of TTM- } \alpha \text { H ONPs doped with different amounts }(0.5 \% \text {, }\end{array}$ \\
\hline
\end{tabular}

$3 \%, 6.5 \%, 13 \%, 26 \%, 50 \%$ in molar ratio) of TTM radical (TTMd-ONPs) were prepared using the re-precipitation method (see SI). The closed-shell TTM- $\alpha \mathrm{H}$ was chosen as the matrix for the ONPs because of its optically neutral behaviour in the range of absorption and emission of the TTM radical. Moreover, its molecular structure, very similar to TTM, shall grant for good solvation of the radical molecules, preventing their aggregation and thus reducing the concentration quenching of the luminescence. The size distribution of the obtained TTMd-ONPs was evaluated via dynamic light scattering (DLS) (see SI). All samples show diameters around $100 \mathrm{~nm}$ (Figure 2) with a polydispersity index lower than 0.22 . Transmission electron microscopy (TEM) images (Figure 2 and Figure S3) confirm the results obtained via DLS and show the spherical morphology of the obtained ONPs. Zpotential measurements (Figure 2) give for all the samples negative surface potential values around $-45 \mathrm{mV}$, which are sufficient to ensure a good colloidal stability of the obtained ONPs aqueous suspensions.

Absorption properties of TTMd-ONPs are quite similar to the ones measured in THF solution, in terms of position and shape of the band. However, the size of the ONPs determines a strong scattering, particularly at wavelength shorter than $400 \mathrm{~nm}$, due to the comparable size of the dispersed nanoparticles.

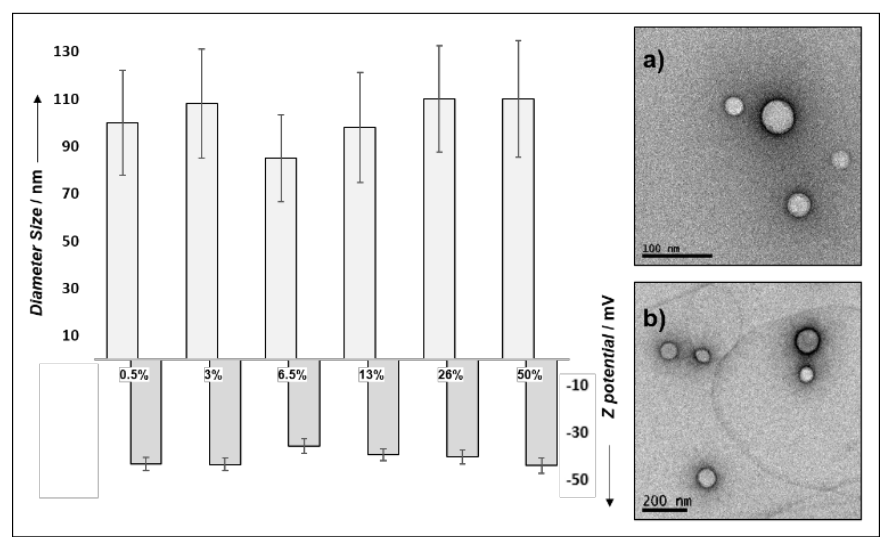

Fig. 2. Left: Size distribution and Z-Potential for TTMd-ONPs (error bars represent the polydispersity). Right: TEM images for a) $0.5 \%$ TTMd-ONPs (100 nm scale), b) $50 \%$ TTMdONPs (200 nm scale).

Correcting the absorption spectra for the scattering contribution was possible using a suspension of pure TTM- $\alpha \mathrm{H}$ nanoparticles with similar size and size distribution as a reference (see SI and Figure S4). This method allows a good estimation of the absorbance, in particular for highly-doped samples. The corrected absorption spectra and the normalized luminescence spectra of TTMd-ONPs at different TTM concentrations are reported in Figure 3, and the main properties are summarized in Table 2. The LQY of the ONPs with low radical doping is strongly improved with respect to the solution. Also not considering the $0.5 \%$ TTMdONPs sample, where the error on the absorbance determination (due to scattering correction) could be significant, it is clear that a rigid environment strongly enhances the emission efficiency giving, at room temperature, values of LQY 10 times higher than in solution. Very interestingly, for percentages of radical-doping equal or higher than $6.5 \%$, dual emission is observed, with a band 
corresponding to the one observed at lower concentrations, and a new broad and structureless band appearing at longer wavelengths, with a maximum shifting from 645 to $685 \mathrm{~nm}$ with increasing radical concentration (Figure 3). This latter band acquires relative intensity with respect to the other one with increasing percentage of radical-doping, while the total LQY decreases (Table 2 and Figure 3 and S5). Self-absorption effects can be ruled both for the monomer emission band (for PTM radicals the $\mathrm{DO} \rightarrow \mathrm{D} 1, \mathrm{HOMO} \rightarrow$ SUMO, transition is almost symmetry-forbidden, with molar absorptivity values lower than 1000 $\mathrm{cm}^{-1} \mathrm{M}^{-1}$ ) and for the excimer emission band (the samples do not absorb at all in that spectral region).
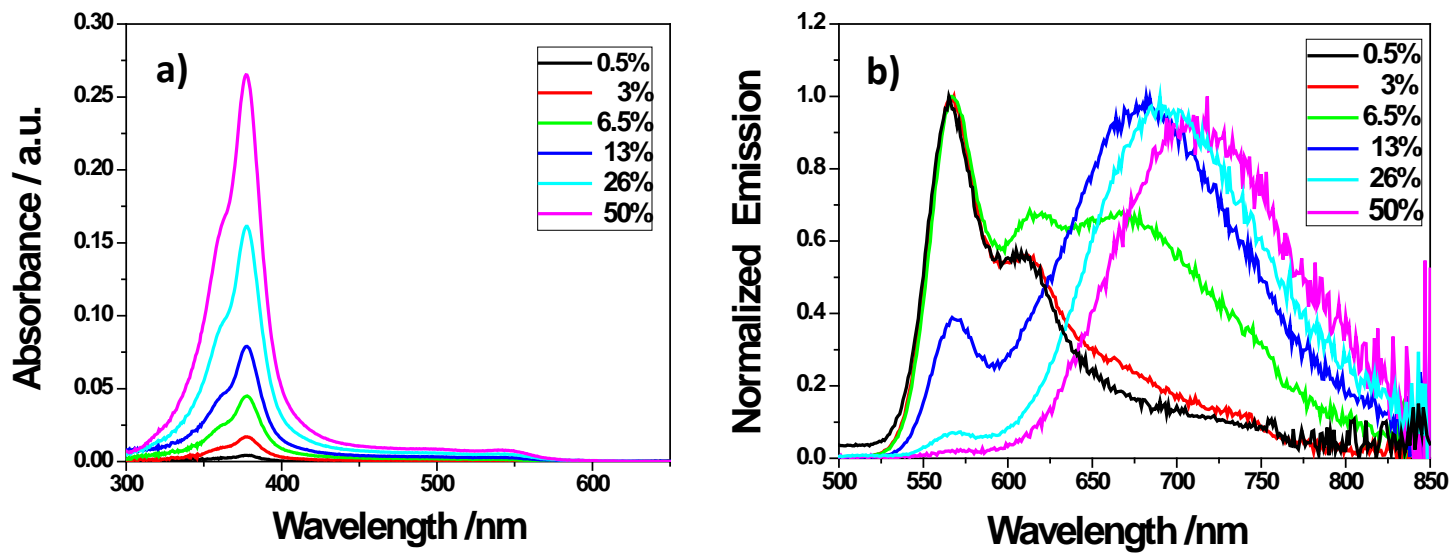

Fig. 3. a) Scattering-corrected absorption spectra of TTMd-ONPs; b) Normalized emission spectra of TTMd-ONPs.
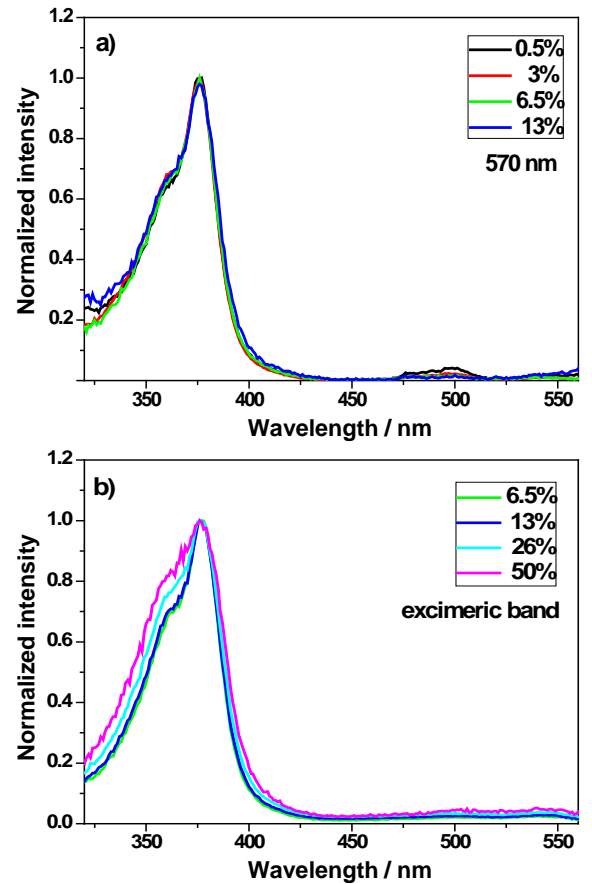

Fig. 4. Normalized fluorescence excitation spectra of TTMd-ONPs registered for emission wavelength fixed at the maximum of the monomer-like emission (a) or at the maximum of the excimer emission (b).

The long-wavelength emission band can be safely ascribed to the formation of excimers of TTM radicals inside the host ONPs. In fact, the absorption spectrum is not affected by the concentration of dopant (Figure 3 ), suggesting a non-interacting ground state, while only emission is affected, as typical of excimers. ${ }^{33,34}$ Moreover, the excitation profile is the same when detecting luminescence on one emission band or the other (Figure 4): this clearly demonstrates that the ground state is dissociated while the excited state can be monomer-like or excimer-like. The excimer is of the "preformed" (or "incipient") type, since diffusion is hindered by the rigid matrix, so that the interacting molecules must already be close before excitation, even if the interaction turns on only when one of the molecules in the dimer is excited. ${ }^{35}$ 
The emission decays measured for the TTMd-ONPs could not be fitted via single-exponential functions, but at least three exponentials were needed (Table S1). Since no direct physical meaning could be associated to the three-exponential fitting, we also performed fittings through a more meaningful function, namely the so-called stretched-exponential function, also known as the Kohlraush-Williams-Watts (KWW) decay function: ${ }^{36}$

$$
I(t)=I_{0} \exp \left[-\left(t / \tau_{0}\right)^{\beta}\right]
$$

Here $\tau_{0}$ is the relaxation time and 6 is the stretch factor, ranging from 0 to 1 . The closer $B$ is to 0 the more the function deviates from a single exponential. The stretched-exponential function is suitable whenever a distribution of decay times or rate constants is expected, as in heterogeneous samples showing continuous lifetime distributions (rigid solutions, samples where energy transfer among like or unlike fluorophores occurs, and so on). ${ }^{37,38,39}$ In particular, the stretched-exponential decay model can provide a direct measure of the heterogeneity of the sample. Typically the parameter $h=1 / 6$ is introduced, so that $h$ increases when the heterogeneity degree increases ( $B$ approaching 0$)$. All the luminescence decays of TTMd-ONPs could be satisfactorily fitted through the stretched-exponential (see Figure S6) function, giving the results reported in Table S1. In Table 1 we report the $h$ values and the average time constants obtained as: $:^{36}$

$$
\langle\tau\rangle=\tau_{0} \Gamma(1+1 / \beta)
$$

where $\Gamma$ indicates the so-called Gamma function:

$$
\Gamma(z)=\int_{0}^{\infty} x^{z-1} e^{x} d x
$$

The average emission lifetimes for the TTM-doped ONPs are always longer than the lifetime obtained in THF solution (Table S1). In particular, the $0.5 \%$-doped TTMd-ONPs have a lifetime of about $220 \mathrm{~ns}$, that is more than one order of magnitude longer than the TTM radical in THF solution ( 10 ns). Increasing the concentration of TTM, we observe a decrease of the lifetime associated to the monomer-like emission. This is not surprising, since the increased TTM concentration increases the number of aggregates and/or incipient excimers, and hence increases the number of potential quenchers for the monomer. ${ }^{40}$

On the contrary, the average lifetime associated to the excimer emission increases slightly more than linearly with the concentration (from $\sim 100$ ns for the 6.5\% TTMd-ONPs to 700 ns for the $26 \%$ TTMd-ONPs), while a decrease is obtained for the most doped sample (50\% TTMd-ONPs, but it should be taken into account that this sample is very weakly emitting, with a LQY on the order of $2 \%$ ). This behaviour can be discussed in relation to the excimer/monomer (E/M) emission ratio, given by (see SI):

$$
\frac{I_{E}}{I_{M}}=\frac{k_{r}^{\prime}}{k_{r}} \boldsymbol{k}_{E} \tau_{E}
$$

where $\boldsymbol{k}_{\boldsymbol{r}}$ and $\boldsymbol{k}_{\boldsymbol{r}}^{\prime}$ are the radiative emission rates of the monomer and of the excimer, respectively; $\boldsymbol{k}_{\boldsymbol{E}}$ is the formation rate of the excimer; $\boldsymbol{\tau}_{\boldsymbol{E}}$ is the emission lifetimes associated to the excimer. Assuming $\boldsymbol{k}_{\boldsymbol{r}}^{\prime} / \boldsymbol{k}_{\boldsymbol{r}}$ as independent of concentration, the E/M emission rate is expected to depend on the fluorophore concentration through the product $\boldsymbol{k}_{\boldsymbol{E}} \boldsymbol{\tau}_{\boldsymbol{E}}$.

In our case, the observed $\mathrm{E} / \mathrm{M}$ emission ratio increases roughly quadratically with respect to the concentration (at least up to the 26\%-doped sample): since a slightly more than linear dependence is accounted for by the excimer lifetime, we expect $\boldsymbol{k}_{\boldsymbol{E}}$ to increase less than linearly with the fluorophore concentration. This is not surprising, since a linear increase of $\boldsymbol{k}_{\boldsymbol{E}}$ with the fluorophore concentration is only expected in the case of diffusion-driven excimer formation.

These results can also be discussed in relation to the specific observed intensities of monomer and excimer emission (see full discussion in the SI). In particular, our data suggest that the (less than linear) increase of $I_{E} / n_{\text {abs }}$ with the concentration up to the 13\%-doping (Figure S5) is mostly governed by $\boldsymbol{k}_{\boldsymbol{E}}$. For higher TTM radical concentration (already starting from the $26 \%$ TTMdONPs) the excimer emission is affected by ACQ, leading to a complete loss of luminescence in the pure TTM samples (either powders or ONPs). 
Table 2. Photophysical properties of TTMd-ONPs and TTMd-PMMA films.

\begin{tabular}{ccccccc} 
& & & \multicolumn{2}{c}{ Mon-like band $^{\mathrm{b}}$} & \multicolumn{2}{c}{ Excimer band $^{\mathrm{c}}$} \\
\cline { 5 - 7 } $\begin{array}{c}\text { ONPs } \\
\text { (\%mol) }\end{array}$ & $\lambda_{\mathrm{em}}{ }^{\mathrm{max}}(\mathrm{nm})$ & LQY $^{\mathrm{a}}$ & $\left\langle\tau_{M}\right\rangle(\mathrm{s})$ & $\mathrm{h}$ & $\left\langle\tau_{E}\right\rangle(\mathrm{s})$ & $\mathrm{h}$ \\
\hline 0.5 & 565 & 0.25 & $2.2 \cdot 10^{-7}$ & 1.6 & - & - \\
3 & 567 & 0.19 & $9.5 \cdot 10^{-8}$ & 1.8 & - & - \\
6.5 & $567 / 645$ & 0.16 & $5.4 \cdot 10^{-8}$ & 2.8 & $1.1 \cdot 10^{-7}$ & 4.0 \\
13 & $567 / 655$ & 0.13 & $8.1 \cdot 10^{-9}$ & 2.6 & $5.4 \cdot 10^{-7}$ & 4.4 \\
26 & $572 / 665$ & 0.07 & $3.0 \cdot 10^{-8}$ & 3.8 & $7.5 \cdot 10^{-7}$ & 1.5 \\
50 & 685 & 0.02 & - & - & $4.5 \cdot 10^{-8}$ & 4.1 \\
Film & & & & & & \\
$(\%$ wt.) & & & & & & \\
1 & 566 & 0.26 & $7.5 \cdot 10^{-8}$ & 1.8 & - & - \\
5 & 572 & 0.09 & $3.6 \cdot 10^{-8}$ & 1.9 & - & - \\
10 & $574 / 734$ & 0.04 & $9.5 \cdot 10^{-9}$ & 2.3 & $8.0 \cdot 10^{-8}$ & 6.6 \\
15 & $572 / 732$ & 0.03 & $1.8 \cdot 10^{-8}$ & 6.1 & $5.9 \cdot 10^{-7}$ & 1.3 \\
20 & $572 / 736$ & 0.02 & $2.5 \cdot 10^{-7}$ & 4.2 & $4.6 \cdot 10^{-7}$ & 3.4 \\
\hline
\end{tabular}

${ }^{a}$ LQY determined using Cresyl Violet perchlorate in $\mathrm{MeOH}(\mathrm{LQY}=0.54)^{41}$ as the reference; the LQY is referred to the total emission, including both emission bands. ${ }^{b / c}$ Mean luminescence lifetimes, $\langle\tau\rangle$, and heterogeneity factor, $h=1 / \beta$, as estimated through stretched-exponential reconvolution fit analysis of the luminescence decay of the monomer-like (b) or excimer-like (c) emission band.

The $h$ value associated to the decay of the monomer-like band increases with increasing radical concentration, suggesting an increased heterogeneity of the monomer-like emissive species for increasing concentration of the emitters themselves. This is consistent with the fact that, for very low radical amount, the emissive radical is mainly surrounded by TTM- $\alpha \mathrm{H}$ host molecules (low heterogeneity) while, for increasing concentration of radical, each TTM molecule can be surrounded by a variable amount of TTM- $\alpha \mathrm{H}$ host molecules or alike TTM radicals (even if weakly interacting), increasing the heterogeneity. The $h$ value associated to the excimer band seems more insensitive to radical concentration, even if a specific trend cannot be recognized (it should be noted, however, that the excimer emission band is partly overlapped to the monomer emission band, so that a contribution from the monomer emission could affect the measured excimer decay).

The rigid environment offered by the TTM- $\alpha \mathrm{H}$ matrix not only helps to obtain good luminescence properties but, thanks to its shielding effect, also contributes to increase the radical photostability, preventing photodegradation processes. The emission intensities of monomeric and excimeric bands of deoxygenated 13\% TTMd-ONPs aqueous suspension were measured as a function of time under continuous irradiation at $375 \mathrm{~nm}$, in order to estimate the photobleaching rate. The results were compared with TTM deoxygenated solution in THF (see Figure 5). The decay of the monomer-like band is characterized by two components: the first one dominating up to $400 \mathrm{~s}$, which matches the decay of TTM in solution, and a second, slower one, sensibly contributing at longer times.

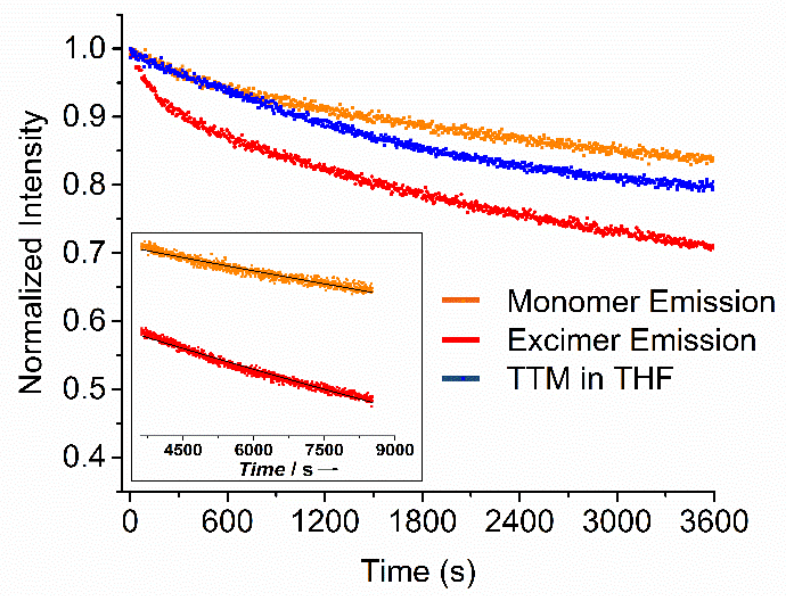

Fig. 5. Luminescence decay of TTM in THF solution (blue points) and 13\% TTMd-ONPs water suspension (monomer-like emission band, orange points; excimer emission band, red points) under continuous irradiation at $375 \mathrm{~nm}$. Inset: luminescence decays of monomeric and excimeric bands of $13 \%$ TTMd-ONPs for time > $1 \mathrm{~h}$, together with the corresponding monoexponential fitting curves (black lines).

Two components can be recognized also in the decay of the excimeric band, but in this case the photobleaching is faster than for TTM in solution, as expected for a dimeric species. The two components in the photobleaching process in ONPs could be due to 
the different environment experienced by TTM molecules on the surface of ONPs with respect to TTM molecules in the inner part of ONPs: more exposed and less hindered the former (leading to faster photodegradation), while completely surrounded by host molecules the latter (leading to slower photodegradation). The decay curves for times longer than 1 hour (i.e. minimizing the effect of the faster photodegradation ascribed to the surface effect) were fitted through mono-exponential curves (inset of Figure 5). The time constant extracted for the monomer-like emission $\left(6.05 \times 10^{5} \mathrm{~s}\right)$ is about twice the time constant estimated for the excimer emission $\left(3.16 \times 10^{5} \mathrm{~s}\right)$, confirming the dimeric nature of the species emitting at long wavelengths.

In order to determine the role played by the specific host matrix in the luminescence behaviour and excimer formation, and to investigate the possible use of these radical-based luminescent materials for optoelectronic devices, TTM-doped (1\%, 5\%, $10 \%, 15 \%, 20 \%$ wt.) PMMA films were prepared via drop-casting of a TTM/PMMA solution in chloroform on quartz substrates (see $\mathrm{SI}$ ). The normalized luminescence spectra of the TTMd-PMMA films are reported in Figure 6 and the main data are summarized in Table 1 (raw spectra in Figure S7). The luminescence behaviour is qualitatively similar to what observed for TTMdONPs, with a monomer-like emission for low radical concentration and a dual emission starting from $10 \%$ radical doping amount. For increasing radical concentration, the relative intensity of the excimer emission increases, while the total LQY is strongly quenched. The excimer emission is centred at $735 \mathrm{~nm}$, i.e. even more to the red with respect to the excimer emission observed for the TTMd-ONPs, and no emission shift with the concentration is observed.

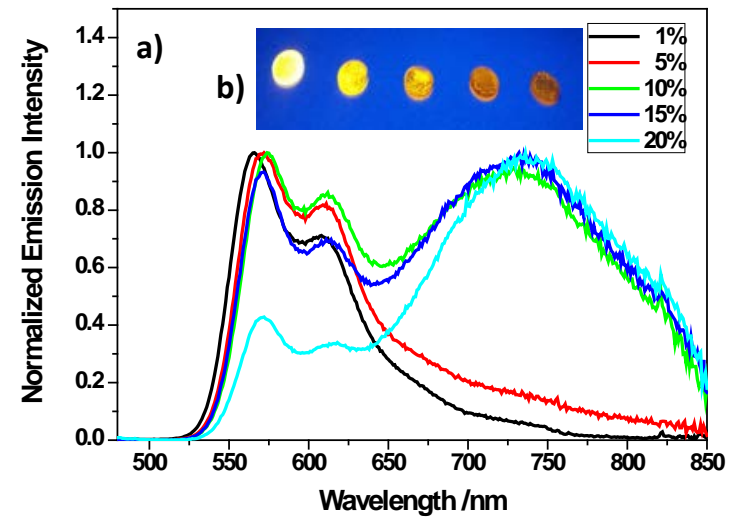

Fig. 6. a) Normalized emission spectra of TTMd-PMMA films with different concentrations of radical. Inset (b): picture of the films under 365 nm illumination (radical concentration increasing from left to right).

As in the case of radical-doped ONPs, luminescence decays could be fitted via either three-exponential or stretched-exponential functions (fitting results in Figure S8 and Table S2). The average time constants extracted from the stretched-exponential fittings are reported in Table 1, together with the values of the $h$ parameter. The behaviour is similar to what reported for radical-doped ONPs: the lifetime associated to the monomer-like emission is lower than in ONPs but decreases for increasing doping, while the lifetime associated to the excimer emission has a non-monotonic trend. Analogously, the $h$ parameter relative to the monomerlike species increases for doping concentration, while the $h$ parameter associated to the excimer behaves non-monotonically. The qualitative (and almost quantitative) similarity with doped-ONPs is impressive, suggesting that the nature of the matrix (small organic molecule vs polymer) does not strongly influence the degree of heterogeneity of the sample.

The $\mathrm{E} / \mathrm{M}$ emission ratio increases roughly linearly with the concentration, suggesting an excimer formation rate barely dependent on the concentration itself. Consistently, $I_{E} / \mathrm{n}_{\text {abs }}$ (Figure $\mathrm{S} 8$ ) barely depends on the TTM concentration.

Although an excimeric emission was observed both in ONPs and in films for high enough radical doping, the position of this emission band and its evolution with the radical concentration are different for the two matrices. In particular, the PMMA doped films show a more red-shifted excimer emission and the position does not depend on the TTM amount, while the excimer emission band of TTM-doped ONPs shifts to the red for increasing doping. The dependence of the excimer emission on the radical concentration is also different in the two types of samples.

These differences can be connected to the different rigidity of the two matrices (Figure S9). In fact, the rigidity and the size of the environmental cage (the volume in which guest molecules are trapped) play a major role in determining the properties of an excimer. ${ }^{42}$ The formation of an excimer requires the presence of a molecular pair that is not favourably interacting in the ground state, but that gains energy once one of the two molecules is excited. Reaching the minimum energy for the excimer implies the relaxation along one or more coordinates, including intermolecular distance (most of all in solution or low-viscosity media), vibrational and conformational modes. Our experimental data suggest that, in a rigid environment as the ONPs, the excimers are trapped in more and more stabilized configurations as the fluorophore concentration increases, with a consequent red-shift of the excimer emission (Figure S9a). ${ }^{43}$ Instead, in a less rigid environment, such as the polymeric films, ${ }^{44}$ relaxation allows to achieve the minimum energy for the excimer, ${ }^{44,45}$ with a consequent concentration-independent excimer emission located at lower energy (Figure S9b). 
The different dependence of the excimer emission intensity on the fluorophore concentration can also be explained accordingly: for "trapped" excimers (as in ONPs), the excimer formation rate depends on the fluorophore concentration; for excimers accessing more degrees of freedom (as in a less rigid polymeric matrix) the excimer formation rate, being dominated by the relaxation step, barely depends on the concentration.

\section{Conclusions}

In conclusion, we demonstrated the possibility to obtain good luminescence quantum yields and long lifetimes, together with an improved photostability in ONPs and polymeric films doped with the carbon-centered free-radical TTM. For increasing radical concentration, a red-shifted, broad and structureless emission band appears, safely attributed to excimers of the TTM radical. In both systems, the lifetime associated to the excimer emission is close to the $\mu$ s timescale, suggesting an almost spin-forbidden emission transition for the excimer species as due, for example, to triplet radical-pairs. ${ }^{46}$ In order to validate this hypothesis further experiment on photo and electro luminescence under magnetic field are in progress. These excimers, observed for the first time in the case of persistent free-radicals, are promising for bioimaging applications as well as for the fabrication of OLEDs with high internal quantum efficiency. Specifically, the TTM-doped ONPs allow to overcome the drawback of water solubility of TTM and increase its biocompatibility ${ }^{47}$ constituting a good example of nano-bioprobes. ${ }^{48-50}$. The peculiar electronic structure of the photoactive radicals provides a perfect scenario for both two-photon excitation and luminescence inside the so-called biological transparency window (namely between 700 and $1500 \mathrm{~nm}$ ), most of all if the excimer emission was exploited. Similarly, the radical-doped polymeric films could be exploited for novel optoelectronic applications, in particular in WOLEDs fabrication, in which blue emitting host materials are doped with low amount of orange/red emitting guest. In this context, the good LQY, the high chemical/photochemical stability offered by the rigid environment, the possibility to cover a wide part of the visible range thanks to the excimer formation and the doublet nature of the emitters, make polychlorotriphenylmethyl radicals a suitable alternative to heavy-metal complexes. ${ }^{51}$

\section{Acknowledgements}

The research leading to these results received funding from the People Programme (Marie Curie Actions) of the European Union's Seventh Framework Programme FP7/2007-2013 under REA grant agreement no. 607721 (ITN Nano2Fun). DGI grant (BeWell; CTQ2013-40480-R and Dynamo MAT2013-50036-EXP), the Networking Research Center on Bioengineering, Biomaterials, and Nanomedicine (CIBER-BBN), the Generalitat de Catalunya (grant 2014-SGR-17) are also acknowledged. ICMAB acknowledges support from the Spanish Ministry of Economy and Competitiveness, through the "Severo Ochoa" Programme for Centres of Excellence in R\&D (SEV- 2015-0496). This work has been developed inside the Material Science PhD programs of Universitat Autonoma de Barcelona and of University of Parma.

\section{Notes and references}

M. Ballester, I. Pascual, C. Carreras and J. Vidal-Gancedo, J. Am. Chem. Soc., 1994, 4205-4210.

I. Ratera and J. Veciana, Chem. Soc. Rev., 2012, 41, 303-349.

M. Souto, M. V. Solano, M. Jensen, D. Bendixen, F. Delchiaro, A. Girlando, A. Painelli, J. O. Jeppesen, C. Rovira, I. Ratera and J. Veciana, Chem. - A Eur. J., 2015, 21, 8816-8825.

J. Guasch, L. Grisanti, V. Lloveras, J. Vidal-Gancedo, M. Souto, D. C. Morales, M. Vilaseca, C. Sissa, A. Painelli, I. Ratera, C. Rovira and J. Veciana, Angew. Chemie - Int. Ed., 2012, 51, 11024-11028.

R. Frisenda, R. Gaudenzi, C. Franco, M. Mas-Torrent, C. Rovira, J. Veciana, I. Alcon, S. T. Bromley, E. Burzurí and H. S. J. Van Der Zant, Nano Lett., 2015, 15, 3109-3114.

L. Yuan, C. Franco, N. Crivilleres, M. Mas-Torrent, L. Cao, C. S. S. Sangeeth, C. Rovira, J. Veciana and C. A. Nijhuis, Nat. Protoc., 2016, 7, 12066.

A. Y. Tesio, D. Blasi, M. Olivares-Marín, I. Ratera, D. Tonti and J. Veciana, Chem. Commun., 2015, 51, 17623-17626.

M. Souto, H. Cui, M. Peña-Álvarez, V. G. Baonza, H. O. Jeschke, M. Tomic, R. Valentí, D. Blasi, I. Ratera, C. Rovira and J. Veciana, J. Am. Chem. Soc., 2016, 138, 11517-11525.

V. Gamero, D. Velasco, S. Latorre, F. López-Calahorra, E. Brillas and L. Juliá, Tetrahedron Lett., 2006, 47, 2305-2309.

L. Fajarí, R. Papoular, M. Reig, E. Brillas, J. L. Jorda, O. Vallcorba, J. Rius, D. Velasco and L. Juliá, J. Org. Chem., 2014, 79, 1771-1777.

S. Castellanos, D. Velasco, F. López-Calahorra, E. Brillas and L. Julia, J. Org. Chem., 2008, 73, 3759-3767.

A. Heckmann, S. Du, J. Pauli, M. Margraf, J. Ko, D. Stich, C. Lambert, I. Fischer, U. Resch-genger and C. Ro, J. Phys. Chem. c, 2009, 113, 20958-20966.

A. Bromberg, K. H. Schmidt and D. Meisel, J. Am. Chem. Soc., 1985, 107, 83-91.

B. B. Craig and M. F. Sonnenschein, J. Lumin., 1989, 43, 227-232. 
A. Bromberg and D. Meisel, J. Phys. Chem., 1985, 89, 2507-2513.

J. A. Schmidt and E. F. Hilinski, J. Am. Chem. Soc., 1988, 110, 4036-4038.

Q. Peng, A. Obolda, M. Zhang and F. Li, Angew. Chemie Int. Ed., 2015, 54, 7091-7095.

Q. Zhang, B. Li, S. Huang, H. Nomura, H. Tanaka and C. Adachi, Nat. Photonics, 2014, 8, 326-332.

M. A. Fox, E. Gaillard and C.-C. Chen, J. Am. Chem. Soc., 1987, 109, 7088-7094.

Y. Hattori, T. Kusamoto and H. Nishihara, Angew. Chemie - Int. Ed., 2015, 54, 3731-3734.

Y. Hattori, T. Kusamoto and H. Nishihara, Angew. Chemie Int. Ed., 2014, 53, 11845-11848.

Y. Hattori, T. Kusamoto and H. Nishihara, RSC Adv., 2015, 5, 64802-64805.

Y. Hattori, T. Kusamoto, T. Sato and H. Nishihara, Chem. Commun., 2016, 440, 908-912.

N. Crivillers, M. Mas-Torrent, S. Perruchas, N. Roques, J. Vidal-Gancedo, J. Veciana, C. Rovira, L. Basabe-Desmonts, B. J. Ravoo, M. Crego-Calama and D. N. Reinhoudt, Angew. Chemie - Int. Ed., 2007, 46, 2215-2219.

C. Simão, M. Mas-Torrent, N. Crivillers, V. Lloveras, J. M. Artés, P. Gorostiza, J. Veciana and C. Rovira, Nat. Chem., 2011, 3, 359364.

F. Vera, M. Mas-Torrent, J. Esquena, C. Rovira, Y. Shen, T. Nakanishi and J. Veciana, Chem. Sci., 2012, 3, 1958.

J. Mei, N. L. C. Leung, R. T. K. Kwok, J. W. Y. Lam and B. Z. Tang, Chem. Rev., 2015, 115, 11718-11940.

B. W. D’Andrade, J. Brooks, V. Adamovich, M. E. Thompson and S. R. Forrest, Adv. Mater., 2002, 14, 1032-1036.

G. M. Farinola and R. Ragni, Chem. Soc. Rev., 2011, 40, 3467-3482.

A. Pucci, F. Di Cuia, F. Signori and G. Ruggeri, J. Mater. Chem., 2007, 17, 783-790.

Y.-H. Chen, K.-C. Tang, Y.-T. Chen, J.-Y. Shen, Y.-S. Wu, S.-H. Liu, C.-S. Lee, C.-H. Chen, T.-Y. Lai, S.-H. Tung, R.-J. Jeng, W.-Y. Hung, M. Jiao, C.-C. Wu and P.-T. Chou, Chem. Sci., 2016, 7, 3556-3563.

A. Jana, L. Bai, X. Li, H. Ågren and Y. Zhao, ACS Appl. Mater. Interfaces, 2016, 8, 2336-2347.

J. B. Birks, Nature, 1967, 214, 1187-1190.

T. Förster, Angew. Chemie Int. Ed., 1969, 8, 333-343.

B. Valeur, Molecular Fluorescence Principles and Applications, 2001, vol. Wiley-VCH.

M. N. Berberan-Santos, E. N. Bodunov and B. Valeur, Chem. Phys., 2005, 315, 171-182.

V. Ptatschek, B. Schreder, K. Herz, U. Hilbert, W. Ossau, G. Schottner, O. Raha, T. Bischof, G. Lermann, A. Materny, W. Kiefer, G. Bacher, A. Forchel, D. Su, M. Giersig, G. Mu and L. Spanhel, J. Phys. Chem. B, 1997, 101, 8898-8906.

A. L. Wong, J. M. Harris and D. B. Marshall, Can. J. Phys., 1990, 68, 1027-1034.

R. Métivier, I. Leray, J.-P. Lefèvre, M. Roy-Auberger, N. Zanier-Szydlowski and B. Valeur, Phys. Chem. Chem. Phys., 2003, 5, 758766.

J. R. Lakowicz, Principles of fluorescence spectroscopy, 2006. Springer Science+Buisness Media, LLC

D. Magde, J. H. Brannon, T. L. Cremers and J. Olmsted, J. Phys. Chem., 1979, 83, 696-699.

J. Kalinowsky, Organic Light-Emitting Diodes: Principles, Characteristics and Processes, 2005.

H. W. Offen and R. R. Eliason, J. Chem. Phys., 1965, 43, 4096-4106.

H. Itagaki, A. Ochiai and T. Nakamori, Eur. Polym. J., 2006, 42, 1939-1952.

R. D. Burkhart and N. Jhon, J. Phys. Chem., 1991, 95, 7189-7196.

N. V Lebedeva, V. F. Tarasov, M. J. E. Resendiz, M. a Garcia-Garibay, R. C. White and M. D. E. Forbes, J. Am. Chem. Soc., 2010, 132, $82-84$.

K. K. Ng and G. Zheng, Chem. Rev., 2015, 115, 11012-11042.

E. Campioli, C. Rouxel, M. Campanini, L. Nasi, M. Blanchard-Desce and F. Terenziani, Small, 2013, 9, 1982-1988.

E. Campioli, D. M. Nikolaidou, V. Hugues, M. Campanini, L. Nasi, M. Blanchard-Desce and F. Terenziani, J. Mater. Chem. C, 2015, 3, 7483-7491.

V. Parthasarathy, S. Fery-Forgues, E. Campioli, G. Recher, F. Terenziani and M. Blanchard-Desce, Small, 2011, 7, 3219-3229.

C. Fan and C. Yang, Chem. Soc. Rev., 2014, 43, 6439-6469. 